\title{
A new simple and reliable vitrification device based on Hollow Fiber Vitrification (HFV) method evaluated using IVP bovine embryos
}

\author{
Galina P. Malenko ${ }^{1}$, Ekaterina V. Kornienko, Ivan I. Nesterov, Gleb Yu Kosovsky \\ Centre of Experimental Embryology and Reproductive Biotechnology, Federal Agency of Scientific Organizations, \\ Moscow, Russia.
}

\begin{abstract}
A new vitrification device based on hollow fiber vitrification (HFV) was constructed using a glass capillary, which lead to simplified construction process and increased practicality of the device. The hollow fiber was attached to heat-pulled tip of the glass capillary using forceps. A protective sheath fitted on the capillary provided protection for the cellulose triacetate hollow fiber with loaded embryos and allowed safe storage in liquid nitrogen for long periods of time (2-12 month), transfer between tanks with liquid nitrogen and transportation within these tanks. No embryos were lost in the process. The device was tested using seven-dayold and eight-day-old IVP bovine blastocysts and expanded blastocysts as a model. Obtained survival (90\% at $24 \mathrm{~h}$ post warming) and hatching rates $(62 \%$ at $72 \mathrm{~h}$ post warming) of day 7 blastocysts and expanded blastocysts were comparable to those gained using various vitrification carriers. Vitrified embryos did not show an increase in the number of cells with damaged membrane or a decrease in total cell number per embryos in comparison to their non-vitrified counterparts. Day 7 and 8 expanded blastocysts did not differ significantly in terms of survival at 24 (97.01 vs. $97.50 \%$ ) and $48 \mathrm{~h}$ post warming (95.52 vs. 95\%), but showed significantly higher survival and hatching rates than day 7 and 8 blastocysts. These results indicated that high and repeatable survival rates can be obtained by selection of IVP bovine embryos at the developmental stage of expanded blastocyst for HFV. Further modification of the method may be required to achieve high and stable results with different developmental stages of IVP bovine embryo. The vitrification device presented in the current article may contribute to wider application of HFV method in livestock production.
\end{abstract}

Keywords: embryo cryopreservation, hollow fiber vitrification, glass capillary device, IVP bovine embryo.

\section{Introduction}

Vitrification is a perspective method of cryopreservation of mammalian embryos and gametes. This method tends to be more effective than slow freezing for cryopreservation of in vitro produced (IVP) mammalian embryos, which are known to be more cryosensitive than embryos obtained in vivo (Nedambale et al., 2004; Saragusty and Arav, 2011). Introduction of the minimal volume cooling (MVC) principle further improved the survival rates after vitrification and led to the emergence of various opentype or surface method carriers based on the principle (Saragusty and Arav, 2011). Yet, vitrification is not routinely used in the livestock production. Despite simplicity of the method, vitrification does require significant experience level of the operator to achieve proper results (Do et al., 2016). Most open-type carriers are not suited for successful cryopreservation of multiple mammalian oocytes or embryos (Kuwayama et al., 2005; Kim et al., 2012) and those that are still require handling of each oocyte or embryo as single object in the vitrification/warming solutions (Park et al., 1999; Kim et al., 2012).

A novel method for simultaneous vitrification of a group of mammalian embryos in cellulose triacetate hollow fibers (hollow fiber vitrification, HFV) was introduced by Matsunari et al. (2012). The method was highly effective for cryopreservation of such cryosensitive objects as in vivo and in vitro produced porcine morulae. Subsequent embryo transfer of these morulae led to the birth of viable offspring (Matsunari et al., 2012; Maehara et al., 2012).

HFV method was initially considered for embryo cryopreservation in polytocous species, as a large number of embryos needed for a single embryo transfer procedure can be vitrified and warmed simultaneously. However, HFV may be also advantageous for preservation of a single embryo of monotocous species such as cow. The handling of an embryo within the hollow fiber in vitrification/warming solutions is significantly simpler and more standardized in comparison to treatment procedures in case of many open-type carriers. HFV method may be also beneficial for groups of IVP bovine embryos as, for instance, several recipients may be prepared simultaneously for practical reasons. HFV may also be used for cryopreservation of bovine oocytes and zona-free embryos produced during handmade cloning, both of which still require a safe and reliable cryopreservation method (Zhou and Li, 2013; Taylor-Robinson et al. 2014).

In livestock production embryos are transferred mainly at the age of 7 days after IVF (Pontes et al., 2010) with the highest pregnancy rates obtained after transfer of day 7 expanded blastocysts (Hasler et al., 1997). Thus, ability of day 7 IVP bovine embryos at different developmental stages to produce steady survival rates after vitrification in hollow fibers holds special interest in regard to the practical application. However, information about effectiveness of bovine embryo vitrification in hollow fibers is limited to 
several abstracts (Beck et al., 2013; Saucedo et al., 2015a,b), and an article concerning cryopreservation of cleavage or morula stage embryos (Uchikura et al., 2016).

Unfortunately, cellulose triacetate hollow fibers have a tendency to become very fragile at low temperatures. To protect hollow fibers from mechanical damage during storage in liquid nitrogen, Matsunari et al. (2012) developed a modified vitrification device. However, authors pointed out that a possible drawback of such device is its' complex structure and complicated construction process. In the following works involving HFV method hollow fibers with vitrified embryos were either warmed immediately (Beck et al., 2013; Saucedo et al., 2015a, b; Uchikura et al., 2016) or placed individually into the cryotubes for storage (Maechara et al., 2012). Simple and safe long-term storage of the samples remained a challenge.

To solve these applicational problems and to make HFV method more practical, the main goal of this article was to introduce a new simple and reliable device based on HFV that allows long term storage of vitrified embryos in liquid nitrogen and can be easily constructed both manually and industrially. The efficiency of the vitrification device was evaluated using day 7 and 8 IVP bovine embryos as a model with special attention to the developmental stage of an embryo.

\section{Materials and Methods}

\section{Construction of the vitrification device}

Present vitrification device was composed of a $2 \mathrm{~cm}$ long piece of a cellulose triacetate hollow fiber (inner diameter $200 \mu \mathrm{m}$, outer diameter $230 \mu \mathrm{m}$, pore size $7 \mathrm{~nm}$; Nipro, Japan), a $7 \mathrm{~cm}$ long glass capillary (outer diameter $1.2 \mathrm{~mm}$; IBI RAS, Russia) with a heat pulled tip and a protective sheath. Outer diameter of the tip varied between 150 and $180 \mu \mathrm{m}$. The piece of hollow fiber was pulled over to the tip of glass capillary for 4-5 mm using forceps (Fig. 1A).

The protective sheath was constructed as follows. A $3.5 \mathrm{~cm}$ long piece of a $0.25 \mathrm{ml}$ cryostraw (Minitube, Germany) was partially inserted (for $2.5 \mathrm{~cm}$ ) into a $5.5 \mathrm{~cm}$ long piece of a $0.5 \mathrm{ml}$ cryostraw. A $0.05 \mathrm{~cm}$ thick, $0.2 \mathrm{~cm}$ wide and $2.5 \mathrm{~cm}$ long polyethylene strip was inserted between contiguous walls of the cryostraws to secure the construction. The piece of $0.25 \mathrm{ml}$ cryostraw was flattened near the open end (Fig. 1B) with a heat sealer. The protective sheath was placed directly over the glass capillary and could be easily moved into "closed" (Fig. 1C) or "open" (Fig. 1D) positions under liquid nitrogen. Additional containers for transfer and identification purposes were constructed from $13 \mathrm{~cm}$ long pieces of a plastic drinking straws (diameter $0.5 \mathrm{~cm}$ ) of different colors heat sealed at one end. On the other end a segment of 3-4 cm in length was cut diagonally.

A

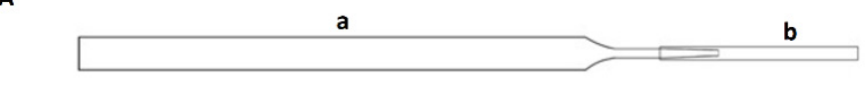

B
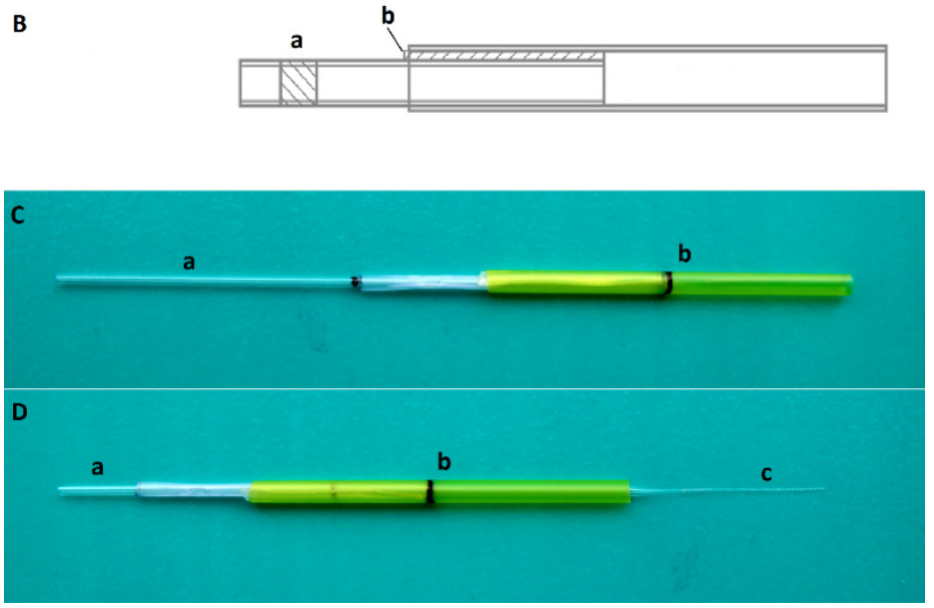

Figure 1. The vitrification device based on cellulose triacetate hollow fiber and glass capillary. A) General scheme of the vitrification device: a) glass capillary with heat-pulled conical tip, b) cellulose triacetate hollow fiber; B) General scheme of the protective sheath made from a piece of $0.25 \mathrm{ml}$ cryostraw and a piece of $0.50 \mathrm{ml}$ cryostraw: a) heat-flattened region of $0.25 \mathrm{ml}$ cryostraw, b) polyethylene strip $0.05 \mathrm{~cm}$ thick; C) Assembled vitrification device in "closed" position: a) glass capillary, b) protective sheath; D) Assembled vitrification device in "open" position: a) glass capillary, b) protectve sheath, c) cellulose triacetate hollow fiber.

\section{Oocyte collection and in vitro maturation}

Unless stated otherwise, all chemicals were obtained from Sigma-Aldrich (USA) and were of embryo tested or cell culture tested quality. All used media were supplemented with $40 \mu \mathrm{g} / \mathrm{ml}$ gentamycin.
Procedures involving oocytes and embryos outside the incubator were carried out at room temperature (22$\left.24^{\circ} \mathrm{C}\right)$.

Bovine ovaries were obtained at a local abattoir and transported to the laboratory in physiological saline solution at $26-30^{\circ} \mathrm{C}$ within $3-5 \mathrm{~h}$. Cumulus-oocyte 
complexes (COCs) were obtained from individual antral follicles (2-8 $\mathrm{mm}$ in diameter) using a dissection device with a single blade (Malenko, 1999). Oocytes with evenly granulated ooplasm and compact or slightly expanded multilayered cumulus were selected for in vitro maturation. Maturation was carried out in TCM199 (PanEco, Russia) supplemented with $0.66 \mathrm{mM}$ of sodium pyruvate, $1 \mathrm{mM}$ L-glutamine, $0.6 \mathrm{mM} \mathrm{L}$ cysteine, $5 \mathrm{IU} / \mathrm{ml}$ chorionic gonadotropin (Moscow endocrine plant FPUE, Russia), $5 \mu \mathrm{g} / \mathrm{ml}$ follicle stimulating hormone (FSH-super, Agrobiomed LLC, Russia), $1 \mu \mathrm{g} / \mathrm{ml}$ of estradiol- $17 \beta$ and $10 \%$ of fetal calf serum (FCS, HyClone, USA). 30-40 COCs were placed into a well of 4-well plate (Nunc, Denmark) containing $0.5 \mathrm{ml}$ of maturation medium and incubated for $22-23 \mathrm{~h}$ in the $\mathrm{CO}_{2}$-incubator at $38.5^{\circ} \mathrm{C}$ and $7 \% \mathrm{CO}_{2}$ in the atmosphere.

\section{In vitro fertilization}

Frozen-thawed bull sperm was used for in vitro fertilization. Spermatozoa were selected by swim-up in TALP-HEPES medium (Bavister and Yanagimachi, 1977; TH). TALP-Fert medium (Parrish et al., 1988; TF) supplemented with $2 \mu \mathrm{g} / \mathrm{ml}$ heparin (Moscow endocrine plant FPUE, Russia) was used for in vitro fertilization. COCs were washed sequentially in $\mathrm{TH}$ for $5 \mathrm{~min}$ and in TF and transferred into a wells of 4-well plate (30-40 COCs per $0.4 \mathrm{ml}$ of TF covered with mineral oil). Then $4 \mu \mathrm{l}$ of PHE solution $(20 \mu \mathrm{m}$ Dpenicillamine, $10 \mu \mathrm{m}$ hypotaurine, $1 \mu \mathrm{m}$ epinephrine) and bull sperm $\left(0.5 \times 10^{6}\right.$ spermatozoa $\left./ \mathrm{ml}\right)$ were added into each well. Oocytes and spermatozoa were incubated for $18-20 \mathrm{~h}$ in the $\mathrm{CO}_{2}$-incubator at $38.5^{\circ} \mathrm{C}$ and $7 \% \mathrm{CO}_{2}$ in the atmosphere. The day of IVF was considered day 0 .

\section{In vitro culture}

Modified SOF medium (Tervit et al., 1972) without BSA or glucose and supplemented with MEM essential and non-essential amino acids and 5\% FCS was used for embryo culture. Presumptive zygotes were denuded of cumulus cells and spermatozoa by vortexing in hyaluronidase solution $(1.1 \mathrm{mg} / \mathrm{ml})$ for $3 \mathrm{~min}$. Then they were washed in modified SOF and placed into wells of 4-well plate, containing $0.4 \mathrm{ml}$ of modified SOF covered with mineral oil. In vitro culture was carried out in the atmosphere containing $5.5 \% \mathrm{CO}_{2}, 6 \%$ $\mathrm{O}_{2}$, and $88.5 \% \mathrm{~N}_{2}$ at $38.5^{\circ} \mathrm{C}$. Developmental stage of the embryos was evaluated on day 7 .

To test the device, morphologically normal day 7 embryos that reached a developmental stage of at least a blastocyst were selected for vitrification and nonvitrification groups. Experiments were repeated at least eight times.

To evaluate the effects of embryo development stage on the outcome of vitrification in hollow fibers morphologically normal day 7 or 8 embryos were selected as follows. On day 7 embryos that reached a developmental stage of at least a blastocyst were removed from the culture. Blastocysts with thinned zona pellucida (about $1 / 3$ of original thickness), significantly larger diameter and compact inner cell mass were considered expanded and selected into one group. The remaining blastocysts were selected into other experimental group. Both groups were vitrified.

The remaining embryos were cultured for another $24 \mathrm{~h}$. Blastocysts and expanded blastocysts that appeared on day 8 were classified as described above and selected into two experimental groups according to their developmental stage. Both groups were vitrified.

\section{Vitrification and warming}

Vitrification and warming were conducted according to Kuwayama et al. (2005) and Matsunari et al. (2012) with minor modifications. In brief, $\mathrm{TH}$ medium (without BSA) supplemented with $20 \%$ FCS (TH20) was used as a base solution. All solutions were at room temperature $\left(22-24^{\circ} \mathrm{C}\right)$. A group of $5-10$ embryos was washed twice in $\mathrm{TH} 20$ and transferred into equilibration solution containing $7.5 \%(\mathrm{v} / \mathrm{v})$ ethylene glycol (EG) and 7.5\% (v/v) dimethyl sulfoxide (DMSO). An aspiration tube was connected to the glass capillary of the vitrification device. The embryos in equilibration solution were aspirated into a hollow fiber. The solution within the hollow fiber containing embryos was separated from the rest of the medium by two air plugs (Fig. 2A). The device was disconnected from the aspiration tube and the capillary was used as a handle for transfer of the hollow fiber between solutions. After incubation in the equilibration solution for $5 \mathrm{~min}$, the hollow fiber with loaded embryos was transferred into vitrification solution containing 15\% EG, 15\% DMSO and $0.5 \mathrm{M}$ sucrose for $60 \mathrm{sec}$ (Fig. 2B) and then was immersed into liquid nitrogen. The protective sheath was "closed" and the entire device was immersed into liquid nitrogen. The devices were either kept under liquid nitrogen for $1 \mathrm{~min}$ before warming or placed into an additional container and inserted into dewar for longtime storage.

Before warming the sheath was "opened". Then the device was removed from liquid nitrogen and the hollow fiber was immediately immersed into warming solution containing $1 \mathrm{M}$ sucrose for $1 \mathrm{~min}$. The tip of the glass capillary was broken off and used as a makeshift "handle" during transfer of the hollow fiber between solutions. The hollow fiber was transferred using forceps into dilution solution with $0.5 \mathrm{M}$ sucrose and incubated for $5 \mathrm{~min}$. Then it was washed twice in TH20 for $5 \mathrm{~min}$, where the embryos were unloaded from the hollow fiber. The embryos were washed in modified SOF medium and cultured for $48 \mathrm{~h}$ (day 8 embryos) or $72 \mathrm{~h}$ (day 7 embryos). Survival and hatching rates of the embryos were evaluated at 24, 48 and $72 \mathrm{~h}$ post warming. Survival rate at $24 \mathrm{~h}$ post warming was determined as a percent of reexpanded embryos with normal morphology from the total number of vitrified embryos. Survival rates at 48 and 72 $\mathrm{h}$ post warming were determined as a percent of nondegraded embryos with normal morphology from the total number of vitrified embryos. Hatching rates were calculated as a percentage from total number of 
vitrified/warmed or non-vitrified embryos.

\section{Cell count}

Average number of cells with damaged membrane per blastocyst was counted as follows. After $24 \mathrm{~h}$ of culture day 7 vitrified/warmed and non-vitrifed embryos were transferred into $0.5 \mathrm{ml}$ of $\mathrm{TH}$ containing $10 \mu \mathrm{g} / \mathrm{ml}$ of propidium iodide and incubated for $5 \mathrm{~min}$ at room temperature in the darkness. Then embryos were transferred into a drop of fresh medium and examined under the fluorescent microscope Nikon Eclipse Ti-U (Nikon, Japan) with corresponding filter (EX 540/25, DM 565, BA 605/55). Number of nuclei with fluorescent signal was counted for each embryo.

Total number of cells was counted as described by Ushijima et al. (2009) with minor modifications. Embryos were placed into hypotonic solution for 5-10 min. Then embryos were fixed in the first fixative (ethanol, acetic acid and distilled water, $3: 2: 1$ ) for 1 minute, and transferred onto a glass slide in the minimum volume drop. A small drop of the second fixative (ethanol and acetic acid, $3: 1$ ) was added to each specimen. Samples were left to dry for $1 \mathrm{~h}$ at room temperature and then were stained with $5 \%$ Giemsa for $1 \mathrm{~h}$ at room temperature. Total number of nuclei in the blastocysts was counted under a microscope at $200 \mathrm{X}$ or 400X magnification.

\section{Statistical analysis}

Survival and hatching rates are presented as corresponding percentages. Results for total cell count in the blastocysts are presented as mean \pm SD. Data was analyzed using chi-square or Student's t-test as appropriate. Significance was set at $\mathrm{P}<0.05$.

\section{Results}

\section{Vitrification device}

A new device based on HFV was developed. Glass capillary served as a base for the whole device and as a supporting frame for a protective sheath. Conical shape of the capillary tip allowed fixation of the hollow fiber without additional measures. Hollow fibers with loaded embryos remained intact within the device during vitrification, storage for 2 to 12 months, transfer between tanks with liquid nitrogen and transportation of these tanks. No embryos were lost in the process. Calculated total volume of the medium containing embryos within the hollow fiber in vitrification solution varied between 0.02-0.04 $\mu$ l (Fig. 2B). Embryos were easily unloaded from the hollow fibers after warming.

The survival rate of day 7 embryos was $90 \%$ at $24 \mathrm{~h}$ after warming and gradually dropped during next $48 \mathrm{~h}$ of culture. Hatching rates at 24,48 and $72 \mathrm{~h}$ post warming were significantly lower in vitrification than in non-vitrification group (Table 1). Average number of cells in the blastocyst did not differ significantly $(\mathrm{P}=$ $0.54)$ between vitrified $(168 \pm 60$ cells, $\mathrm{n}=27)$ and nonvitrified $(178 \pm 65$ cells, $\mathrm{n}=30)$ groups after $24 \mathrm{~h}$ of culture. No significant difference was found between average numbers of cells with damaged membranes either (3.43 vs. 2.86 cells per blastocyst, correspondingly).

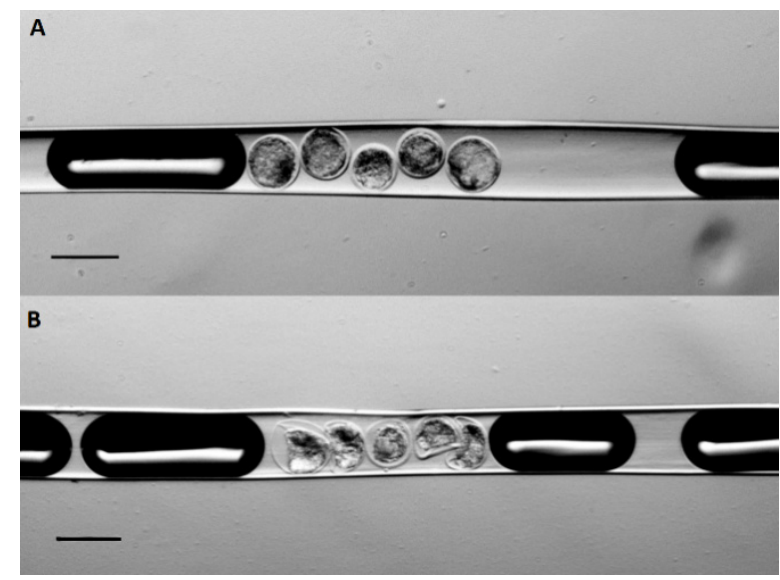

Figure 2. IVP bovine blastocysts loaded into a cellulose triacetate hollow fiber. A) Loaded blastocysts in the equilibration solution containing 7.5\% of EG and 7.5\% DMSO; B) Loaded blastocysts transferred within the hollow fiber into vitrification solution containing $0.5 \mathrm{M}$ sucrose, $15 \%$ of EG and 15\% DMSO. Scale is equal $200 \mu \mathrm{m}$.

Table 1. Survival and hatching rates of day 7 IVP bovine embryos vitrified in cellulose triacetate hollow fibers

\begin{tabular}{|c|c|c|c|c|c|c|c|}
\hline \multirow[t]{3}{*}{ Group } & \multirow{3}{*}{$\begin{array}{l}\text { No. of } \\
\text { embryos }\end{array}$} & \multicolumn{6}{|c|}{ Hours post warming (h) } \\
\hline & & \multicolumn{2}{|c|}{24} & \multicolumn{2}{|c|}{48} & \multicolumn{2}{|c|}{72} \\
\hline & & Survived (\%) & Hatched (\%) & Survived (\%) & Hatched $(\%)$ & Survived (\%) & Hatched $(\%)$ \\
\hline Vitrification & 150 & $135(90)$ & $24\left(16^{\mathrm{a}}\right)$ & $125(83.33)$ & $76\left(50.67^{\mathrm{a}}\right)$ & 115 (76.67) & $93\left(62^{a}\right)$ \\
\hline Non-vitrification & 81 & $81(100)$ & $33\left(40.74^{\mathrm{b}}\right)$ & $81(100)$ & $56\left(71.60^{\mathrm{b}}\right)$ & $81(100)$ & $69\left(85.19^{b}\right)$ \\
\hline
\end{tabular}

$\overline{\mathrm{a}, \mathrm{b}}$ percentages with different superscripts within the same column differ significantly $(\mathrm{P}<0.05)$. 
Vitrification of IVP bovine blastocysts and expanded blastocysts

Average diameter of blastocysts and expanded blastocysts prior to vitrification was 168 and $187 \mu \mathrm{m}$, correspondingly. Survival rate of vitrified day 7 expanded blastocysts was $97.01 \%$ at $24 \mathrm{~h}$ post warming. Hatching rate at $72 \mathrm{~h}$ was $94.54 \%$. Survival and hatching rates of expanded blastocysts were significantly higher at all time points than corresponding rates of blastocysts (Table 2). Day 7 expanded blastocysts had on average more cells in total $(192 \pm 56$ cells $)$ and less cells with damaged membrane (3.42) at $24 \mathrm{~h}$ post warming than day 7 blastocysts (144 \pm 56 and 5.17, correspondingly). However, the results did not differ significantly.

The survival rates of vitrified day 8 expanded blastocysts at $24(97.50 \%)$ and $48 \mathrm{~h}$ post warming $(95 \%)$ were significantly higher than those obtained for day 8 blastocysts ( 52.78 and $41.67 \%$, correspondingly). Same tendency was observed for corresponding hatching rates (Table 2).

Table 2. Survival and hatching rates of day 7 and 8 IVP bovine blastocysts and expanded blastocysts vitrified in cellulose triacetate hollow fibers.

\begin{tabular}{|c|c|c|c|c|c|c|c|c|}
\hline \multirow{3}{*}{$\begin{array}{l}\text { Age of } \\
\text { embryos }\end{array}$} & \multirow[t]{3}{*}{ Developmental stage } & \multirow{3}{*}{$\begin{array}{l}\text { No. of } \\
\text { embryos }\end{array}$} & \multicolumn{6}{|c|}{ Hours post warming $(\mathrm{h})$} \\
\hline & & & \multicolumn{2}{|c|}{24} & \multicolumn{2}{|c|}{48} & \multicolumn{2}{|c|}{72} \\
\hline & & & Survived (\%) & Hatched $(\%)$ & Survived (\%) & $\begin{array}{l}\text { Hatched } \\
(\%)\end{array}$ & $\begin{array}{l}\text { Survived } \\
(\%)\end{array}$ & $\begin{array}{l}\text { Hatched } \\
(\%)\end{array}$ \\
\hline \multirow[t]{2}{*}{ day 7} & Blastocyst & 70 & $50\left(71.43^{\mathrm{a}}\right)$ & $5\left(7.14^{\mathrm{a}}\right)$ & $49\left(70^{\mathrm{a}, \mathrm{c}}\right)$ & $29\left(41.43^{\mathrm{a}, \mathrm{c}}\right)$ & $47\left(67.14^{\mathrm{a}}\right)$ & $40\left(57.14^{\mathrm{a}}\right)$ \\
\hline & Expanded blastocyst & 67 & $65\left(97.01^{\mathrm{b}}\right)$ & $28\left(41.79^{b}\right)$ & $64\left(95.52^{\mathrm{b}}\right)$ & $50\left(74.63^{b}\right)$ & $64\left(95.52^{b}\right)$ & $62\left(94.54^{\mathrm{b}}\right)$ \\
\hline \multirow[t]{2}{*}{ day 8} & Blastocyst & 36 & $19\left(52.78^{\mathrm{a}}\right)$ & $1\left(2.78^{\mathrm{a}}\right)$ & $15\left(41.67^{\mathrm{a}, \mathrm{d}}\right)$ & $5\left(13.89^{\mathrm{a}, \mathrm{d}}\right)$ & - & - \\
\hline & Expanded blastocyst & 40 & $39\left(97.50^{b}\right)$ & $15\left(37.50^{b}\right)$ & $38\left(95.00^{\mathrm{b}}\right)$ & $27\left(67.50^{b}\right)$ & - & - \\
\hline
\end{tabular}

\footnotetext{
a,b percentages with different supperscripts within the same column and the same day differ significantly $(\mathrm{P}<0.05)$.

${ }^{\mathrm{c}, \mathrm{d}}$ percentages with different supperscripts within the same column differ significantly $(\mathrm{P}<0.05)$.
}

\section{Discussion}

A new simple and practical vitrification device based on HFV is presented in the current article. The construction of the device is based on $1.2 \mathrm{~mm}$ thick glass capillary with a heat-pulled tip. One of the advantages of the glass capillary is that it is available, practical and easily disposable material. The tip of the capillary can be easily prepared both industrially for mass production and manually in the laboratory for experimental purposes. Conical shape of the tip allows secure fixation of the hollow fiber without any additional measures (Matsunari et al., 2012), which significantly simplifies construction process. Glass capillary also serves as a supporting frame for a protective sheath, which can be easily "closed" or "opened" under liquid nitrogen. The sheath protected hollow fiber with loaded embryos during storage in liquid nitrogen and no fibers were lost in the process despite their fragility. Due to relative compactness of the device, it can be placed easily into additional container such as conventional goblet or a suggested above container, which allows relocation of individual devices between tanks with liquid nitrogen and easier identification. Placement and storage of the device required no additional precaution and attention from the operator unlike the situation when hollow fibers were stored in the standard cryotubes (Maehara et al., 2012; Matsunari et al., 2012).

The device was tested using day 7 bovine embryos as a model. The survival rate $(90 \%)$ in the experimental group at $24 \mathrm{~h}$ post warming was comparable to those obtained after group vitrification of
IVP bovine embryos using hollow fibers (Beck et al., 2013) and various open-type carriers (Abdalla et al., 2010; Kim et al., 2012). The highest survival rates of the bovine embryos (100\%) were obtained using McGill Cryoleaf (Huang et al., 2007) and Cryotech (Gutnisky et al., 2013).

The survival rate of the vitrified/warmed embryos declined during $72 \mathrm{~h}$ of culture, and hatching rates were significantly lower compared to non-vitrified embryos. However, a total of 93 out of 115 surviving blastocysts $(80.85 \%)$ hatched after $72 \mathrm{~h}$ of culture (Table 1). Total cell count per blastocyst did not differ significantly between the groups $(168 \pm 60 v s .178 \pm 65$ cells for vitrified and non-vitrified embryos, respectively). Unlike data shown in some of the works (Shirazi et al., 2009), there was also no significant increase in the average number of cells with damaged membrane per blastocyst in the vitrification group (3.43 cells) compared to the non-vitrification group (2.86). Obtained results indicate that the surviving blastocysts may retain their viability after vitrification in hollow fibers.

When day 7 embryos were divided before vitrification according to their developmental stage, expanded blastocysts showed significantly higher survival rate $(97.01 \%)$ than blastocysts $(71.43 \%)$. The same tendency was observed for embryos that developed into expanded blastocysts or blastocysts on day 8 (97.50 vs. 52.78\%, correspondingly). Hatching rates of the expanded blastocysts were also significantly higher for both day 7 and 8 embryos. Day 7 vitrified blastocysts had a tendency to have more cells with damaged membrane (5.17) in comparison to vitrified 
expanded blastocysts (3.42). However, the results did not differ significantly.

Obtained results are in agreement with data shown in literature (Han et al., 1994; Hasler et al., 1997; Dinnyes et al., 1999; Rios et al., 2010), as it is generally expected for morphologically normal and more developmentally advanced embryos on the given day to be of higher quality and have higher viability after cryopreservation. It was also shown that day 7 blastocysts and expanded blastocysts survived cryopreservation better than their slower developing day 8 and 9 counterparts (Han et al., 1994; Saha et al., 1996; Dinnyes et al., 1999; Gomez et al., 2008). Contrary to this, in our experiment there was no significant difference in survival and hatching rates found between day 7 and 8 expanded blastocysts at 24 and $48 \mathrm{~h}$ post warming. These results are in agreement with Abdalla $e t$ al. (2010). Moreover, in our case the survival rate of day 7 and 8 expanded blastocysts shows no significant decline during culture unlike tendency shown by day 7 and 8 blastocysts. These results may indicate that expanded blastocysts have morphological and physiological traits that allow them to survive cryopreservation better than earlier developmental stages as suggested by Dinnyes et al. (1999). Stage dependent ability to ensure cryopreservation is indirectly confirmed by survival rate of day 9 hatched bovine blastocysts vitrified in hollow fibers $(23 / 23$ embryos survived $24 \mathrm{~h}$ post warming; Malenko et al., 2016, CEERB FASO, Russia; unpublished data). The effect may be correlated with general lower quality of IVP bovine morulae and blastocysts compared to in vivo produced ones due to abundance of lipid droplets in the cytoplasm and less developed junctional complexes between trophoblast cells (Abe et al., 1999). At the same time, concentration of lipid droplets was shown to decline after morula stage in both bovine and porcine in vivo produced embryos (Abe et al., 1999; Romek et al., 2009). This tendency may be present in IVP embryos during further development making later stages such as expanded or hatched blastocyst less sensitive to suboptimal cryopreservation conditions. There are, however, also data showing that expanded bovine blastocysts produced both in vitro (Shirazi et al., 2009) and in vivo (Hasler, 2011), are more susceptible to cryoinjury than early blastocysts, which correlates with the trait observed in other mammalian species (Vanderzwalmen et al., 2002; Mukaida et al., 2003; Li et al., 2012).

There are numerous discrepancies in the protocols used by different laboratories making direct comparison of the results quite difficult. For example, the division of the embryos according to their developmental stage is highly subjective (Dinnyes et al., 1999; Gomez et al., 2008; Abdalla et al., 2010). However, one of the major factors that may contribute to the differences in the survival rates of vitrified bovine embryos of different developmental stage and age seems to be the choice of the either surface type carriers based on MVC principle (Huang et al., 2007; Abdalla et al., 2010; Gutnisky et al., 2013) or tubing carriers (Dinnyes et al., 1999; Gomez et al., 2008; Rios et al.,
2010). Thus, our preliminary data obtained using Cryotop carrier showed leveling of the difference in survival rates of day 7 IVP bovine blastocysts (17/18 embryos) and expanded blastocysts (15/15 embryos). No significant difference in the survival rates was also found between day 7 or 8 expanding ( 85 and $84 \%$ ) and fully expanded blastocysts ( 88 and $89 \%$ ) vitrified using Cryotop (Abdalla et al. (2010) and maximum survival rates of the mixed population of day 7 bovine blastocysts $(100 \%)$ were obtained using close analogues of this carrier (Huang et al., 2007; Gutnisky et al., 2013).

Minimum volume cooling principle is also utilized in HFV method. In our case, volume of the solution within the hollow fiber containing 5-10 embryos varied between 0,02 and $0,04 \mu \mathrm{l}$ after incubation in vitrificatin solution. However, in comparison to open-type carriers embryos are separated from vitrification and warming solutions by a cellullose triacetate film. The thickness of the fiber wall is comparable to thickness of zona pellucida $(15 \mu \mathrm{m})$. Eventhough the pores in the hollow fibers are relatively large $(7 \mathrm{~nm})$, the rate of cryoprotectant redistribution between solutions in and out of the hollow fiber might be affected. This may lead to suboptimal vitrification/warming conditions crucial for the survival of more sensitive part of the embryo population. Thus, the HFV method would show lower survival rates for mixed population of day 7 IVP bovine embryos compared to Cryologic method (Saucedo et al., 2015a, b). At the same time, the developmental rate of pig parthenogenetic morulae obtained using HFV method was significantly higher compared to the one obtained using the Cryotop method (Nagashima et al., 2015). In the current state, high and repeatable survival rates after HFV can be obtained using expanded and possibly hatched IVP bovine blastocysts. The procedures, however, should be modified to accommodate embryos at the earlier stages of blastocoel expansion.

In conclusion, a new simple, compact and reliable device for HFV was constructed and tested using day 7 and 8 IVP bovine embryos as a model. The device was based on a glass capillary and supplied with a protective sheath. It was proven to be reliable and practical for vitrification and long term storage of the IVP bovine embryos in liquid nitrogen. Presented device may contribute to wider application of HFV method in livestock production for both politocous and monotocous species leading to significantly simplified and standardized vitrification and storage procedures. Mixed group of day 7 IVP bovine blastocysts and expanded blastocysts vitrified using the device showed survival rates comparable to those obtained using other vitrification carriers. Day 7 and 8 expanded blastocysts survived vitrification significantly better than blastocysts of the same age. Currently, high and repeatable results with HFV method can be obtained by selection of IVP bovine embryos at the developmental stage of expanded blastocyst. However, the ability of such embryos to successfully implant and produce viable offspring must be verified in further experiments. 
Further modification of the method may be required to achieve high and stable results with different developmental stages of IVP bovine embryos.

\section{References}

Abdalla H, Shimoda M, Hara H, Morita H, Kuwayama M, Hirabayashi M, Hochi S. 2010. Vitrification of ICSI- and IVF-derived bovine blastocysts by minimum volume cooling procedure: effect of developmental stage and age. Theriogenology, 74:1028-1035.

Abe H, Yamashita S, Itoh T, Satoh T, Hoshi H. 1999. Ultrastructure of bovine embryos developed from in vitro-matured and -fertilized oocytes: comparative morphological evaluation of embryos cultured either in serum-free medium or in serum-supplemented medium. Mol Reprod Dev, 53:325-335.

Bavister BD, Yanagimachi R. 1977. The effects of sperm extracts and energy sources on the motility and acrosome reaction of hamster spermatozoa in vitro. Biol Reprod, 16:228-237.

Beck A, Kurome M, Nagashima H, Reichenbach M, Reichenbach HD, Wolf E. 2013. Hollow fiber vitrification of biopsied in vitro produced bovine blastocysts. Reprod Biol, 13(suppl. 2):57.

Dinnyes A, Lonergan P, Fair T, Boland MP, Yang X. 1999. Timing of the first cleavage post-insemination affects cryosurvival of in vitro-produced bovine blastocysts. Mol Reprod Dev, 53:318-324.

Do VH, Walton S, Catt S, Taylor-Robinson AW. 2016. Requirements for cryopreservation of in vitroproduced bovine embryos by a standard method of vitrification. J Vet Sci Anim Husb, 3:1-8.

Gomez E, Rodriguez A, Munoz M, Caamano JN, Hidalgo CO, Moran E, Facal N, Diez C. 2008. Serum free embryo culture medium improves in vitro survival of bovine blastocysts to vitrification. Theriogenology, 69:1013-1021.

Gutnisky C, Alvarez GM, Cetica PD, Dalvit GC. 2013. Evaluation of the cryotech vitrification kit for bovine embryos. Cryobiology, 67:391-393.

Han YM, Yamashina H, Koyama N, Lee KK, Fukui Y. 1994. Effects of quality and developmental stage on the survival of IVF-derived bovine blastocysts cultured in vitro after freezing and thawing. Theriogenology, 42:645-654

Hasler JF, Hurtgen PJ, Jin ZQ, Stokes JE. 1997. Survival of IVF-derived bovine embryos frozen in glycerol or ethylene glycol. Theriogenology, 46:563-579.

Hasler JF. 2011. Effect of embryo stage on pregnancy rate following direct transfer of bovine embryos frozen in ethylene glycol. Reprod Fertil Dev, 24:131.

Huang JY, Chung JT, Tan SL, Chian RC. 2007. High survival and hatching rates following vitrification of embryos at blastocyst stage: a bovine model study. Reprod Biomed Online, 14:464-470.

Kim YM, Uhm SJ, Gupta MK, Yang JS, Lim J-G, Das ZC, Heo YT, Chung H-J, Kong I-K, Kim N-H, Lee HT, Ko DH. 2012. Successful vitrification of bovine blastocysts on paper container. Theriogenology, 78:1085-1093.
Kuwayama M, Vajta G, Ieda S, Kato O. 2005. Comparison of open and closed methods for vitrification of human embryos and the elimination of potential contamination. Reprod Biomed Online, 11:608-614.

Li L, Zhang X, Zhao L, Xia X, Wang W. 2012. Comparison of DNA apoptosis in mouse and human blastocysts after vitrification and slow freezing. Mol Reprod Dev, 79:229-236.

Maehara M, Matsunari H, Honda K, Nakano K, Takeuchi Y, Kanai T, Matsuda T, Matsumura Y, Hagiwara Y, Sasayama N, Shirasu A, Takahashi M, Watanabe M, Umeyama K, Hanazono Y, Nagashima H. 2012. Hollow fiber vitrification provides a novel method for cryopreserving in vitro maturation/fertilization-derived porcine embryos. Biol Reprod, 87:133, 1-8.

Malenko GP. 1999. Device to recover bovine cumulusoocyte complexes (COCs) without destroying their cumulus layers from antral ovarian follicles. In:

Proceedings 15th Meeting European Embryo Transfer Association, Lyon. Paris: AETE. pp. 198.

Matsunari H, Maehara M, Nakano K, Ikezawa Y, Hagiwara Y, Sasayama N, Shirasu A, Ohta H, Takahashi M, Nagashima H. 2012. Hollow fiber vitrification: a novel method for vitrifying multiple embryos in a single device. J Reprod Dev, 58:599-608.

Mukaida T, Nakamura S, Tomiyama T, Wada S, Oka C, Kasai M, Takahashi K. 2003. Vitrification of human blastocysts using cryoloops: clinical outcome of 223 cycles. Hum Reprod, 18:384-391.

Nagashima H, Uchikura A, Maehara M, Hatae S, Nakano K. 2015. Direct comparison of the hollow-fiber vitrification and conventional vitrification methods. In: IFFS/JSRM International Meeting, 2015, Yokohama, Japan. Mt. Royal, NJ: IFFS. (abstract).

Nedambale TL, Dinnyes A, Groen W, Dobrinsky JR, Tian XC, Yang X. 2004. Comparison on in vitro fertilized bovine embryos cultured in KSOM or SOF and cryopreserved by slow freezing or vitrification. Theriogenology, 62:437-449.

Park SP, Kim EY, Kim DI, Park NH, Won YS, Yoon SH, Chung KS, Lim JH. 1999. Simple, efficient and successful vitrification of bovine blastocysts using electron microscope grids. Hum Reprod, 14:2838-2843.

Parrish JJ, Susko-Parrish JL, Winer MA, First NL. 1988. Capacitation of bovine sperm by heparin. Biol Reprod, 38:1171-1180.

Pontes JHF, Silva KCF, Basso AC, Rigo AG, Ferreira CR, Santos GMG, Sanches BV, Porcionato JPF, Vieira PHS, Faifer FS, Sterza FAM, Schenk JL, Seneda MM. 2010. Large-scale in vitro embryo production and pregnancy rates from Bos taurus, Bos indicus, and indicus-taurus dairy cows using sexed sperm. Theriogenology, 74:1349-1355.

Rios GL, Mucci NC, Kaiser GG, Alberio RH. 2010. Effect of container, vitrification volume and warming solution on cryosurvival of in vitro-produced bovine embryos. Anim Reprod Sci, 118:19-24.

Romek M, Gajda B, Krzysztofowicz E, Smorg Z. 2009. Lipid content of non-cultured and cultured pig embryo. Reprod Domest Anim, 44:24-32. 
Saha S, Rajamahendran R, Boediono A, Sumantril C, Suzuki T. 1996. Viability of bovine blastocysts obtained after 7, 8 or 9 days of culture in vitro following vitrification and one-step rehydration. Theriogenology, 46:331-343.

Saragusty J, Arav A. 2011. Current progress in oocyte and embryo cryopreservation by slow freezing and vitrification. Reproduction, 141:1-19.

Saucedo MN, Kurome M, Reichenbach M, Wolf E, Reichenbach HD. 2015a. Cryopreservation of bovine in vitro-produced embryos: intrinsic factors determining vitrification outcomes. Reprod Fertil Dev, 27(suppl. 1):120.

Saucedo MN, Reichenbach M, Kurome M, Wolf E, Reichenbach HD. 2015b. Vitrification of intact and splitted in vitro produced d7 bovine embryos. Anim Reprod, 12:858.

Shirazi A, Nazari H, Ahmadi E, Heidari B, ShamsEsfandabadi N. 2009. Effect of culture system on survival rate of vitrified bovine embryos produced in vitro. Cryobiology, 59:285-290.

Taylor-Robinson AW, Walton S, Swain DL, Walsh KB, Vajta G. 2014. The potential for modification in cloning and vitrification technology to enhance genetic progress in beef cattle in Northern Australia. Anim Reprod Sci, 148:91-96.

Tervit HR, Whittingham DG, Rowson LEA. 1972. Successful culture in vitro of sheep and cattle ova. $J$ Reprod Fertil, 30:493-497.

Uchikura A, Matsunari H, Nakano K, Hatae S, Nagashima H. 2016. Application of hollow fiber vitrification for cryopreservation of bovine early cleavage stage embryos and porcine morulablastomeres. J Reprod Dev, 62:219-223.

Ushijima H, Akiyama K, Tajima T. 2009. Classification of morphological changes based on the number of cleavage divisions in bovine embryos. $J$ Reprod Dev, 55:83-87.

Vanderzwalmen P, Bertin G, Debauche CH, Standaert V, van Roosendaal E, Vandervorst $M$, Bollen M, Zech H, Mukaida T, Takahashi K, Schoysman R. 2002. Births after vitrification at morula and blastocyst stages: effect of artificial reduction of the blastocoelic cavity before vitrification. Hum Reprod, 17:744-751.

Zhou GB, Li N. 2013. Bovine oocytes cryoinjury and how to improve their development following cryopreservation. Anim Biotechnol, 24:94-106. 